\title{
Flood risk assessment by a multicriteria spatial analysis in the municipalities of Pobè and Adja-Ouèrè
}

\section{Evaluation du risque d'inondation par une analyse spatiale multicritère dans les communes de Pobè et d'Adja-Ouèrè}

\author{
Akibou A. AKINDELE ${ }^{\text {let } 2}$, Lionel TODOME ${ }^{1}$
}

${ }^{1}$ Département de Géographie et Aménagement du Territoire (Centre Universitaire d'Adjarra).

${ }^{2}$ Laboratoire Pierre PAGNEY : Climats, Eau, Ecosystèmes et Développement, Université d'Abomey-Calavi 03 BP 1122 Jéricho, Cotonou 03 (Rép. Du Bénin) Email : akybson@yahoo.fr et ltodome@gmail.com

Received: 21 Feb 2021; Received in revised form: 29 Apr 2021; Accepted: 12 May 2021; Available online: 29 May 2021

(C)2021 The Author(s). Published by Infogain Publication. This is an open access article under the CC BY license

(https://creativecommons.org/licenses/by/4.0/).

\begin{abstract}
Floods are natural phenomena that give rise to a wave of interest in the management of space especially following their recurrences and intensifications. In order to prevent them, it is essential to carry out risk assessment studies.For this purpose, several methods of flood risk assessment and modeling are used. Of all, combination of Geographic Information Systems (GIS) and Multicriteria Analysis guarantees considerable accuracy in results obtained. This methodology combines risk identification and spatialization of results using the WLC algorithm to perform complex calculations on each pixel. Hazard criteria (soil types, slope, rainfall, altitude, groundwater potential and water accumulation) and vulnerability criteria (land use, degree of poverty and population density) were identified, standardized, weighted and aggregated during the analysis based on Hierarchical Analysis Process and Weighted Linear Combination algorithm. Weighted superposition of hazard and vulnerability criteria maps reveals four categories of flood risk areas. These are areas with a low risk of flooding (17.69\%), areas with medium flood risk (50.28\%), areas with high risk of flooding (27.39\%) and areas with very high flood risk (4.64\%).Thus, $32.03 \%$ of the study area presents a significant flood risk, precisely in the districts of Kpoulou, Ahoyeye, Igana, Issaba, Adja-Ouèrè and Massè. A significant portion of the 18,000 households in these boroughs are at risk of flooding, which threatens human lives and socio-economic activities.
\end{abstract}

Keywords-Risk, Flood, Geographic Information Systems (GIS), Multicriteria Analysis, Analytical Hierarchical Process (AHP), Weighted Linear Combination (WLC).

Résumé-Les inondations sont des phénomènes naturels qui suscitent une vague d'intérêt dans la gestion de l'espace notamment suite à leurs récurrences et intensifications. Afin de les prévenir il s'avère primordial d'effectuer des études d'évaluations de risque. A cet effet, plusieurs méthodes d'évaluation et de modélisation des risques d'inondation sont de mises. De toutes, la combinaison des Systèmes d'Informations Géographiques (SIG) et de l'Evaluation Multicritère (EMC)garantie une précision considérable dans les résultats obtenus. Cette méthodologie allie des fonctionnalités d'identification du risque et la spatialisation des résultats au moyen de l'algorithme WLC qui effectue des calculs complexes sur chaque pixel. Les critères d'aléa (types de sols, pente, pluviométrie, altitude, potentialité en eau 
souterraine et accumulation d'eau) et les critères de vulnérabilité (utilisation du sol, degré de pauvreté et densité de la population) ont été identifiés, standardisés, pondérés et agrégés au cours de l'analyse en se basant sur leProcessus d'Analyse Hiérarchique et l'algorithme de Combinaison Linéaire Pondérée. La superposition pondérée des cartes critères aléa et vulnérabilité permet de mettre en évidence quatre catégories d'espaces à risque d'inondation. Il s'agit des espaces à faible risque d'inondation (17,69\%), des espaces au risque d'inondation moyen (50,28\%), des espaces au risque élevé d'inondation (27,39\%) et des espaces au risque d'inondation très élevé (4,64\%). Ainsi, 32,03\% du secteur d'étude présente un risque d'inondation important, précisément dans les arrondissements de Kpoulou, Ahoyéyé, Igana, Issaba, AdjaOuèrè et Massè. Une importante partie des 18.000 ménages présents dans ces arrondissements sont exposés aux risques d'inondation qui menacent vies humaines et activités socioéconomiques.

\section{Mots clés-Risque, Inondation, Système d'Information Géographique (SIG), Evaluation Multicritère (EMC), Processus d'Analyse Hiérarchique, Combinaison Linéaire Pondérée.}

\section{INTRODUCTION}

Les phénomènes naturels en particulier les inondations ont depuis toujours fait partie du quotidien des humains dans certaines régions du globe. De plus en plus récurrents à cause de plusieurs facteurs, ces phénomènes engendrent toujours plus de dégâts matériels et humains à chaque survenance. Le continent africain étant l'un des plus exposés aux phénomènes d'inondations, il s'avère plus qu'urgent d'initier plusieurs études dans le but de prévoir ces phénomènes naturels et d'amoindrir les conséquences néfastes qui en découlent.

Etant considérée comme une des régions les plus pauvre de la planète, l'Afrique de l'Ouest dont fait partie le Bénin est souvent confronté à des phénomènes climatiques extrêmes. Ce niveau de pauvreté élevé, accentue la vulnérabilité face à ces phénomènes en ce sens que moins la population dispose de moyens et de capacités d'adaptation face à un risque élevé, plus la vulnérabilité est grande (Wallez L., 2010, p5).

En 2010, le Bénin a subi les pires inondations de son histoire avec les $2 / 3$ de son territoire touché et plus des dégâts matériels estimés selon le Rapport d'évaluation des Besoins Post Catastrophe $^{1}$ (2011, p12) à 78,3 milliards de FCFA (près de 160 millions USD).Cet évènement a mis en avant la nécessité de multiplier les actions à caractère prévisionnels sur les inondations. A cet effet, plusieurs articles scientifiques et initiatives gouvernementales ont été produites dans l'optique d'une prévision plus efficace des phénomènes d'inondations au Bénin. Faisant appel à plusieurs méthodes, les différents articles publiés n'intègrent pas toujours tous les facteurs et toutes les spécificités propres à chacune des régions béninoises.

${ }^{1}$ INONDATION AU Bénin - Rapport d'évaluation des Besoins Post Catastrophe 2011, 84p :

https://www.gfdrr.org/sites/gfdrr/files/GFDRR Benin PD NA 2010.pdf
Pour ce présent article, il est question de faire appel à une méthodologie combinant les SIG et l'analyse spatiale multicritère afin d'évaluer le risque d'inondation dans le secteur d'étude. L'intégration des SIG et des méthodes d'analyse multicritère constitue une voie privilégiée et incontournable pour faire évoluer les SIG vers de véritables systèmes d'aide à la décision.

Cette approche a déjà été appliquée par plusieurs auteurs l'estimation de l'aptitude d'un site ou la modélisation du risque d'inondation (C. Kêdowidé, 2010, p1 ; C Kubalet al., 2009, p.1884; D. Riconet al., 2018, p.2 ; M.Safaripouret al., 2012, p1818). L'utilisation de cette méthode précise, donne des orientations sur les politiques de prévision des inondations, la mise en place d'une culture du risque et la réduction des dégâts qui en découlent dans les zones concernées.

\section{ZONE D'ETUDE}

Le secteur d'étude prend en compte les communes de Pobè et Adja-Ouèrè situées dans le département du plateau au Sud-Est du Bénin. Couvrant une superficie de $863 \mathrm{Km}^{2}$, le secteur d'étude est situé entre $6^{\circ} 51^{\prime} 20^{\prime \prime}$ ' et $7^{\circ} 12^{\prime} 22^{\prime \prime}$ ' de latitude Nord et entre $2^{\circ} 30^{\prime} 13^{\prime}$ ' et $2^{\circ} 46^{\prime} 26^{\prime}$ ' de longitude Est. Le climat y est de type subéquatorial avec deux saisons sèches et deux saisons pluvieuses avec des hauteurs pluviométriques varient entre 1100 et $1200 \mathrm{~mm}$ par an.

Par ailleurs, le réseau hydrographique du secteur d'étude est faiblement significatif avec quelques cours d'eau temporaires. Situé globalement dans une zone de plateaux de 50 à 200 mètres au-dessus du niveau de la mer et d'une altitude moyenne de 100 mètres, le secteur d'étude comporte une dépression qui n'est rien d'autre que la continuité de la dépression médiane qui traverse tout le Bénin d'Ouest à Est.

Estimée à 239. 959 habitants (INSAE, RGPH4, 2013), la population du secteur d'étude est composée en majorité de 
holli et de nago, s'adonnant majoritairement aux activités agricoles.

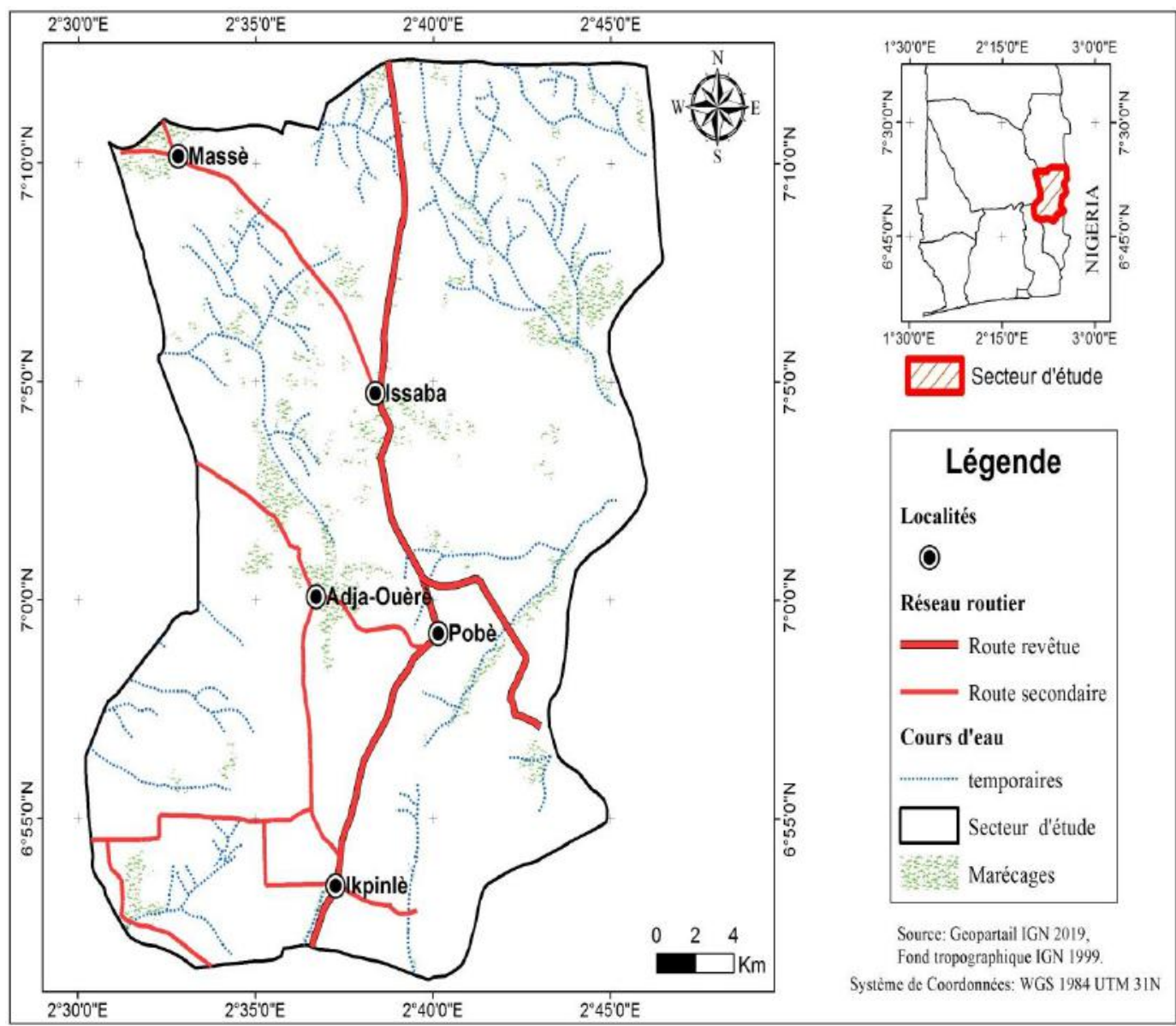

Fig.1 : Carte de situation géographique du secteur d'étude

\subsection{Matériel}

\section{MATERIEL ET METHODES}

Pour ce travail, le matériel est constitué d'un GPS, un appareil photo-numérique, un drone, un ordinateur, de Microsoft Excel, des logiciels SIG (Qgis 3.0 et ArcGIS 10.3) et du logiciel Envi. Les différentes données utilisées, leurs caractéristiques et sources figurent dans le tableau I.

Tableau I : Données utilisées, caractéristiques et sources

\begin{tabular}{|l|l|l|l|}
\hline \multirow{2}{*}{$\begin{array}{l}\text { Images } \\
\text { Satellites }\end{array}$} & Sentinel 2A & $\begin{array}{l}\text { Résolution spatiale }(10 \mathrm{~m}-20 \mathrm{~m}-60 \mathrm{~m}) \text { du } \\
01 / 01 / 2019 .\end{array}$ & usgs \\
\cline { 2 - 4 } & DEM & Résolution spatiale 30m & usgs \\
\hline Cartes & $\begin{array}{l}\text { Cartes topographique, pédologique, hydrographique, } \\
\text { hydrogéologique }\end{array}$ & $\begin{array}{l}\text { IGN, CENAP, GIZ- } \\
\text { DGEAU }\end{array}$ \\
\hline Données démographiques & $\begin{array}{l}\text { INSAE } \\
\text { Données pluviométriques }\end{array}$ & Travaux de terrain \\
\hline Informations issues des enquêtes de terrain &
\end{tabular}

Source : Akindélé et al., 2019 


\subsection{Méthodes}

Dans le cadre de cette étude, le couple SIG-EMC a été utilisé. L'évaluation multicritère a consisté en un choix des critères d'évaluation, la standardisation des facteurs, la pondération des facteurs par la matrice de Saaty (1990, p15) et de l'agrégation au moyen de la superposition pondérée dans le logiciel SIG.

\subsubsection{Choix des critères d'évaluation}

Etant la première étape fondamentale pour l'évaluation du risque d'inondation, les critères constituent le socle sur lequel repose ce travail de recherche. A cet effet, une attention particulière a été accordée à cette partie. Le choix des critères utilisés pour cette évaluation du risque d'inondation se réfère à la bibliographie et aux spécificités du secteur d'étude. Ainsi, les critères ont été choisis en fonction du type de cartographie à effectuer : cartographie de l'aléa inondation et cartographie de la vulnérabilité.

\subsubsection{Cartographie de l'aléa inondation}

Afin de cartographier l'aléa inondation les critères suivants ont été retenus: il s'agit des types de sols, la pente, la pluviométrie,l'altitude, la potentialité en eau souterraine et l'accumulation d'eau.

La prédisposition des sols à l'inondation est fonction de la perméabilité et de la capacité de rétention des différents types de sols présents dans le secteur d'étude. La carte critère sols est obtenue par une rastérisation et une reclassification des sols en fonction des propriétés desdits sols.

La pente est l'un des facteurs les plus importants intervenant dans la cartographie du risque d'inondation. A cet effet toutes les études précédentes en tiennent compte. Elle est calculée automatiquement puis reclassifiée en cinq classes (très faibles, faibles, moyennes, fortes et très fortes) en fonction de la valeur de la pente.

La pluviométrie fait partie des paramètres influençant grandement les inondations. En effet, un excès de précipitation sur une période donnée provoque un écoulement très lent des eaux et engendre un ruissellement de forte envergure. Ainsi, une forte précipitation favorise une susceptibilité élevée à l'inondation. La carte isohyète est obtenue par interpolationdu total pluviométrique mensuelle durant la grande saison pluvieuse (début Avril-Mi-Juillet) au moyen de la méthode IDW. Les données pluviométriques de quatre stations ont été recueillies et épurées à cet effet.

Les tranches d'altitude influent sur les inondations en ce sens que les zones les plus basses sont les plus susceptibles à être inondé. La carte des tranches d'altitudes est extraite de la reclassification du modèle numérique d'altitude en cinq classes (très faibles, faibles, moyennes, fortes et très fortes).

La potentialité en eau souterraine est dégagée en se référant à la carte hydrogéologique du Bénin. Les zones aux potentiels hydrogéologiques élevés se voient attribuer les scores les plus élevés.

Les zones d'accumulation d'eau constituent naturellement des unités cartographiques où l'eau est présente à la surface. En cas de pluviométrie excessive, les alentours de ces zones sont les premiers inondés. La carte d'accumulation des eaux est réalisée à partir de l'outil Arc hydro.

La standardisation des critères est effectuée sur la base d'une échelle de 0 à 10 choisie en fonction de l'échelle d'évaluation du logiciel et des données disponibles.

L'attribution des scores est faite en se basant sur les résultats de la revue bibliographique.Les classes susceptibles de favoriser l'aléa inondation sont affectées des scores les plus élevés.

Tableau II : standardisation des critères d'aléa

\begin{tabular}{|c|c|c|}
\hline Critère & Classe & Score \\
\hline \multirow[t]{4}{*}{ Types de sols } & Sols ferralitiques & 2 \\
\hline & Sols ferrugineux & 3 \\
\hline & Vertisols & 8 \\
\hline & Sols hydromorphes & 9 \\
\hline \multirow[t]{5}{*}{ Pente $(\%)$} & Très forte & 2 \\
\hline & Forte & 3 \\
\hline & Moyenne & 5 \\
\hline & Faible & 8 \\
\hline & Très faible & 10 \\
\hline \multirow{5}{*}{$\begin{array}{l}\text { Pluviométrie } \\
(\mathrm{mm})\end{array}$} & $<520$ & 4 \\
\hline & $520-530$ & 5 \\
\hline & $530-540$ & 6 \\
\hline & $540-550$ & 7 \\
\hline & $>550$ & 8 \\
\hline \multirow[t]{5}{*}{ Altitude (m) } & $>160$ & 2 \\
\hline & $120-160$ & 3 \\
\hline & $80-120$ & 4 \\
\hline & $40-80$ & 5 \\
\hline & $<40$ & 8 \\
\hline \multirow{2}{*}{$\begin{array}{l}\text { Potentialité en } \\
\text { eau souterraine }\end{array}$} & Faible à limitée & 1 \\
\hline & $\begin{array}{l}\text { Médiocre } \\
\text { moyenne }\end{array}$ & 4 \\
\hline
\end{tabular}




\begin{tabular}{|l|l|c|}
\hline & favorable & 6 \\
\hline $\begin{array}{l}\text { Accumulation } \\
\text { d'eau }\end{array}$ & Non & 0 \\
\cline { 2 - 3 } & Oui & 10 \\
\hline
\end{tabular}

Source : Akindélé et al., 2019

\subsubsection{Cartographie de la vulnérabilité}

La cartographie de la vulnérabilité prend en compte les critères : l'utilisation du sol, le degré de pauvreté et la densité de la population.

La densité de la population est un critère de vulnérabilité en ce sens que les zones les plus densément peuplées sont plus vulnérables aux catastrophes et phénomènes naturels que celles sui ne le sont pas. En se référant aux statistiques de la population, la carte de densité de la population dans chaque arrondissement du secteur d'étude a été réalisée.

Etant dans une zone prédominée par les activités agricoles pratiquées de façon traditionnelles, le secteur d'étude fait partie d'une zone faiblement développée. Toutefois, le degré de pauvreté est inégalement réparti dans l'espace ; variant d'un arrondissement à un autre. Les zones les plus pauvres et ne disposant d'infrastructure d'accueil de sinistrés ou d'évacuations sont les plus vulnérables. La cartographie du degré de pauvreté est réalisée de façon participative avec la collaboration des autorités locales.A cet effet, trois classes sont définies (faible, moyen et élevé). Le score de pondération augmente au fur et à mesure que le degré de pauvreté s'accroit.

L'utilisation du sol est un critère de vulnérabilité du fait de la nature du type d'utilisation ou d'occupation. En cas d'inondation, les effets et dégâts sont nettement plus considérables dans les agglomérations, les usines et les zones agricoles.

L'attribution des scores est faite en se basant sur les résultats des enquêtes de terrain et de la revue bibliographique.Les classes susceptibles d'être les plus vulnérables à l'inondation sont affectées des scores les plus élevés.
Tableau III : standardisation des critères de vulnérabilité

\begin{tabular}{|l|l|l|}
\hline Critère & Classe & Score \\
\hline \multirow{4}{*}{ Densité de la population } & Faible & 3 \\
\cline { 2 - 3 } & Moyenne & 5 \\
\cline { 2 - 3 } Degré de pauvreté & Elevé & 7 \\
\hline \multirow{5}{*}{ Utilisation du sol } & Faible & 3 \\
\cline { 2 - 3 } & Moyen & 5 \\
\cline { 2 - 3 } & Elevé & 7 \\
\hline \multirow{5}{*}{} & Marécages & 2 \\
\cline { 2 - 3 } & Sol nu & 2 \\
\cline { 2 - 3 } & Plantations & 2 \\
\cline { 2 - 3 } & $\begin{array}{l}\text { Champs, Cultures et } \\
\text { Jachère }\end{array}$ & 8 \\
\cline { 2 - 3 } & Agglomérations & 10 \\
\hline
\end{tabular}

Source : Akindélé et al., 2019

\subsubsection{Pondération des critères}

Plusieurs méthodes existent pour le choix du poids. On retrouve notamment la comparaison par paire, qui consiste à évaluer tous les critères par paire et à les trier par ordre d'importance dans un tableau. Cette méthode utilisant le Processus d'Analyse Hiérarchique ou Analytical Hierarchy Process(AHP) développée par Saaty (1990, p12) a été utilisée à cet effet.

Elle permet de produire des coefficients de pondération standardisés dont la somme est égale à « $100 \%$ ou 1 ». Le poids des facteurs indique leur importance relative par rapport à tous les autres. Les poids des facteurs sont déterminés à partir d'une série de comparaison par paire de ces critères en tenant compte de l'importance relative de deux critères à l'aléa et à la vulnérabilité. Leur importance est arrangée dans une matrice de décision ou matrice de comparaison par paire (tableau IV) et déterminée sur une échelle numérique d'importance à 9 niveaux (tableau $\mathrm{V}$ ).

Tableau IV : Matrice de comparaison par paire (Saaty, 1990, p12)

\begin{tabular}{|l|l|l|l|l|}
\cline { 2 - 5 } \multicolumn{1}{c|}{} & $\mathrm{C} 1$ & $\mathrm{C} 2$ & $\ldots$ & $\mathrm{Cn}$ \\
\hline $\mathrm{C} 1$ & $\mathbf{1} 2$ & $\mathrm{a}_{12}$ & & $\mathrm{a}_{1 \mathrm{n}}$ \\
\hline$\ldots$ & $\mathrm{a}_{21}=1 / \mathrm{a}_{12}$ & $\mathbf{1}$ & & $\mathrm{a}_{2 \mathrm{n}}$ \\
\hline $\mathrm{Cn}$ & & & $\mathbf{1}$ & \\
\hline
\end{tabular}

Source : Saaty, 1990, p12. 
En effet quand $a_{i j}$ représente le jugement quantitatif de la paire de critères $C_{i}, C_{j}$, il est défini par les règles suivantes :

1. $\mathrm{Si} \mathrm{a}_{\mathrm{ij}}=\mathrm{x}$, alors $\mathrm{a}_{\mathrm{ji}}=1 / \mathrm{x}$

2. Si Ci est considérée comme étant d'une importance relative égale à $\mathrm{Cj}$, alors $\mathrm{a}_{\mathrm{ij}}=1, \mathrm{a}_{\mathrm{ji}}=1$ et $\mathrm{a}_{\mathrm{ii}}=1$, pour tout i.

Une fois que la matrice de comparaison est remplie, il est procédé au calcul du vecteur propre de chaque critère et du vecteur propre normalisé lui correspondant. Le vecteur propre indique l'ordre de priorité ou la hiérarchie des caractéristiques étudiées.

Ce résultat est important pour l'évaluation de la probabilité, puisqu'il sera utilisé pour indiquer l'importance relative de chaque critère opérant. Le vecteur propre est la mesure qui permettra d'évaluer la cohérence ou la qualité de la solution obtenue, représentant ainsi, un autre avantage de cette méthode. Le vecteur propre de la matrice $\left(\mathrm{W}_{\mathrm{i}}\right)$ peut être trouvé grâce à la formule suivante :

$$
W_{i}=\left(\prod_{i=1}^{n} a_{i j}\right)^{1 / n}
$$

En outre, celui-ci doit être normalisé afin que la somme de ses éléments soit égale à l'unité. Pour cela, il suffit de calculer la proportion de chaque élément par rapport à l'addition (soit la fréquence).

$\mathrm{T}_{1}=\left(\mathrm{W} 1 / \sum \mathrm{Wi}\right) \times 100$

$\mathrm{T}_{2}=\left(\mathrm{W} 2 / \sum \mathrm{Wi}\right) \times 100$

$\mathrm{Tn}=\left(\mathrm{Wn} / \sum \mathrm{Wi}\right) \times 100$

Avec $\mathrm{T}$ le vecteur propre normalisé encore appelé coefficient de pondération utilisé pour quantifier et évaluer l'importance de chaque critère.

Tableau V: Echelle numérique d'importance à 9 niveaux

\begin{tabular}{|l|l|l|}
\hline $\begin{array}{l}\text { Degrés } \\
\text { d'importance }\end{array}$ & Définition & Explication \\
\hline 1 & Importance égale & $\begin{array}{l}\text { Deux caractéristiques contribuent de la même façon à } \\
\text { l'objectif. }\end{array}$ \\
\hline 3 & $\begin{array}{l}\text { Faible importance d'une caractéristique } \\
\text { par rapport à une autre }\end{array}$ & $\begin{array}{l}\text { L'expérience et l'appréciation personnelle favorisent } \\
\text { légèrement une caractéristique par rapport à une autre. }\end{array}$ \\
\hline 5 & Importance forte ou déterminante & $\begin{array}{l}\text { L'expérience et l'appréciation favorisent fortement une } \\
\text { caractéristique par rapport à une autre. }\end{array}$ \\
\hline 7 & Importance très forte ou attestée & $\begin{array}{l}\text { Une caractéristique est fortement favorisée et sa } \\
\text { dominance est attestée dans la pratique. }\end{array}$ \\
\hline $2,4,6,8$ & $\begin{array}{l}\text { Importance absolue paleurs associées à des jugements } \\
\text { intermédiaires }\end{array}$ & $\begin{array}{l}\text { Les preuves favorisant une caractéristique par rapport à } \\
\text { une autre sont aussi convaincantes que possible. }\end{array}$ \\
\hline
\end{tabular}

Source : Adapté de Saaty (1990, p15)

L'application de l'AHP aux critères identifiés dans le cadre de cette recherche permet d'obtenir les matrices de comparaison par paire pour l'aléa et la vulnérabilité.

Tableau VI : Matrice de comparaison par paire des critères (Aléa)

\begin{tabular}{|l|c|c|c|c|c|c|}
\cline { 2 - 7 } \multicolumn{1}{l|}{} & $\begin{array}{l}\text { Types de } \\
\text { Sols }\end{array}$ & Pente & Altitude & $\begin{array}{l}\text { Pluviom } \\
\text { étrie }\end{array}$ & $\begin{array}{l}\text { Potentiel en eau } \\
\text { souterraine }\end{array}$ & $\begin{array}{l}\text { Accumulation des } \\
\text { eaux }\end{array}$ \\
\hline Types de Sols & $\mathbf{1}$ & & & & & \\
\hline Pente & $1 / 3$ & $\mathbf{1}$ & & & & \\
\hline Altitude & $1 / 3$ & $1 / 3$ & $\mathbf{1}$ & & & \\
\hline Pluviométrie & $1 / 5$ & $1 / 5$ & $1 / 3$ & 1 & $1 / 5$ & $\mathbf{1}$ \\
\hline $\begin{array}{l}\text { Potentiel en eau } \\
\text { souterraine }\end{array}$ & $1 / 5$ & $1 / 5$ & $1 / 5$ & $1 / 7$ & $\mathbf{1}$ & \\
\hline $\begin{array}{l}\text { Accumulation des } \\
\text { eaux }\end{array}$ & $1 / 7$ & $1 / 7$ & $1 / 7$ & $\mathbf{1}$ & $\mathbf{0 , 2 3}$ \\
\hline Wi & $\mathbf{3 , 4 1}$ & $\mathbf{2 , 3 7}$ & $\mathbf{1 , 5 1}$ & $\mathbf{0 , 8 8}$ & $\mathbf{0 , 4 1}$ & $\mathbf{3} \%$ \\
\hline Ti & $\mathbf{3 8} \%$ & $\mathbf{2 7} \%$ & $\mathbf{1 7} \%$ & $\mathbf{1 0} \%$ & $\mathbf{5 \%}$ & \\
\hline
\end{tabular}

Source : Akindélé et al., 2019 
Tableau VII : Matrice de comparaison par paire des critères (Vulnérabilité)

\begin{tabular}{|l|c|c|c|}
\cline { 2 - 4 } \multicolumn{1}{c|}{} & Utilisation du sol & Degré de pauvreté & Densité de la population \\
\hline Utilisation du sol & $\mathbf{1}$ & $\mathbf{1}$ & \\
\hline Degré de pauvreté & 1 & $1 / 3$ & $\mathbf{1}$ \\
\hline Densité de la population & $1 / 3$ & $\mathbf{1 , 4 4}$ & $\mathbf{0 , 4 8}$ \\
\hline Wi & $\mathbf{1 , 4 4}$ & $\mathbf{4 3 \%}$ & $\mathbf{1 4} \%$ \\
\hline Ti & $\mathbf{4 3 \%}$ & & \\
\hline
\end{tabular}

Source : Akindélé et al., 2019

$\mathrm{Au}$ terme de la pondération l'agrégation est réalisée. Elle consiste à multiplier chaque couche-facteur par son coefficient de pondération et ensuite à additionner ces résultats pour produire un indice de risque situé sur une échelle prédéterminée $(0$ 10). A cet effet, l'agrégation au moyen de la méthode de superposition pondérée est utilisée.

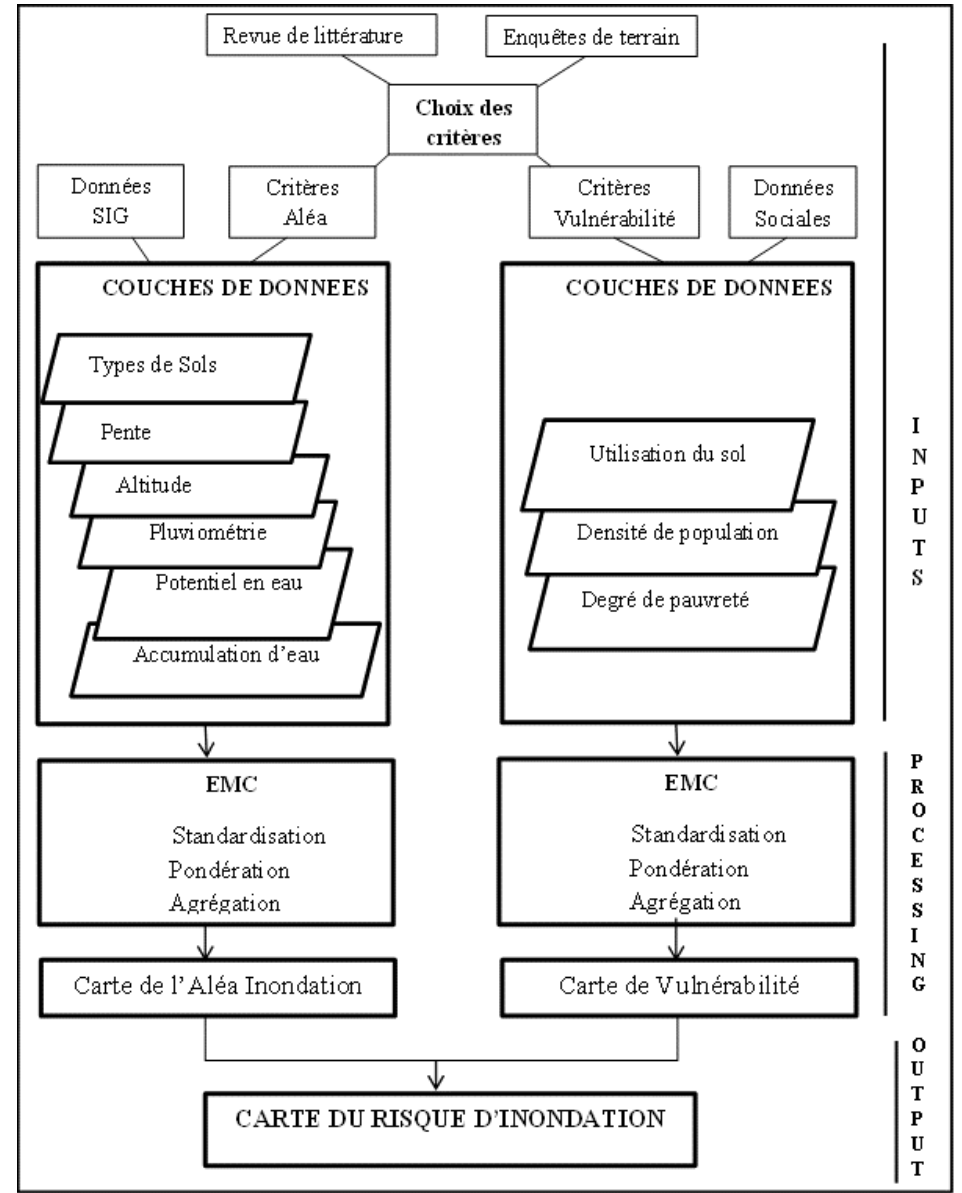

Fig.2: Diagramme méthodologique de la cartographie multicritère du risque d'inondation

\subsection{Cartographie de l'aléa inondation}

\section{RESULTATS}

En prélude à la l'obtention de la carte de l'aléa inondation, les cartes pondérées des différents critères sont réalisées. L'agrégation des cartes critères (format raster) conduit à la carte de l'aléa. La planche 1 présente les différentes cartes critères réalisées pour la cartographie de l'aléa. 

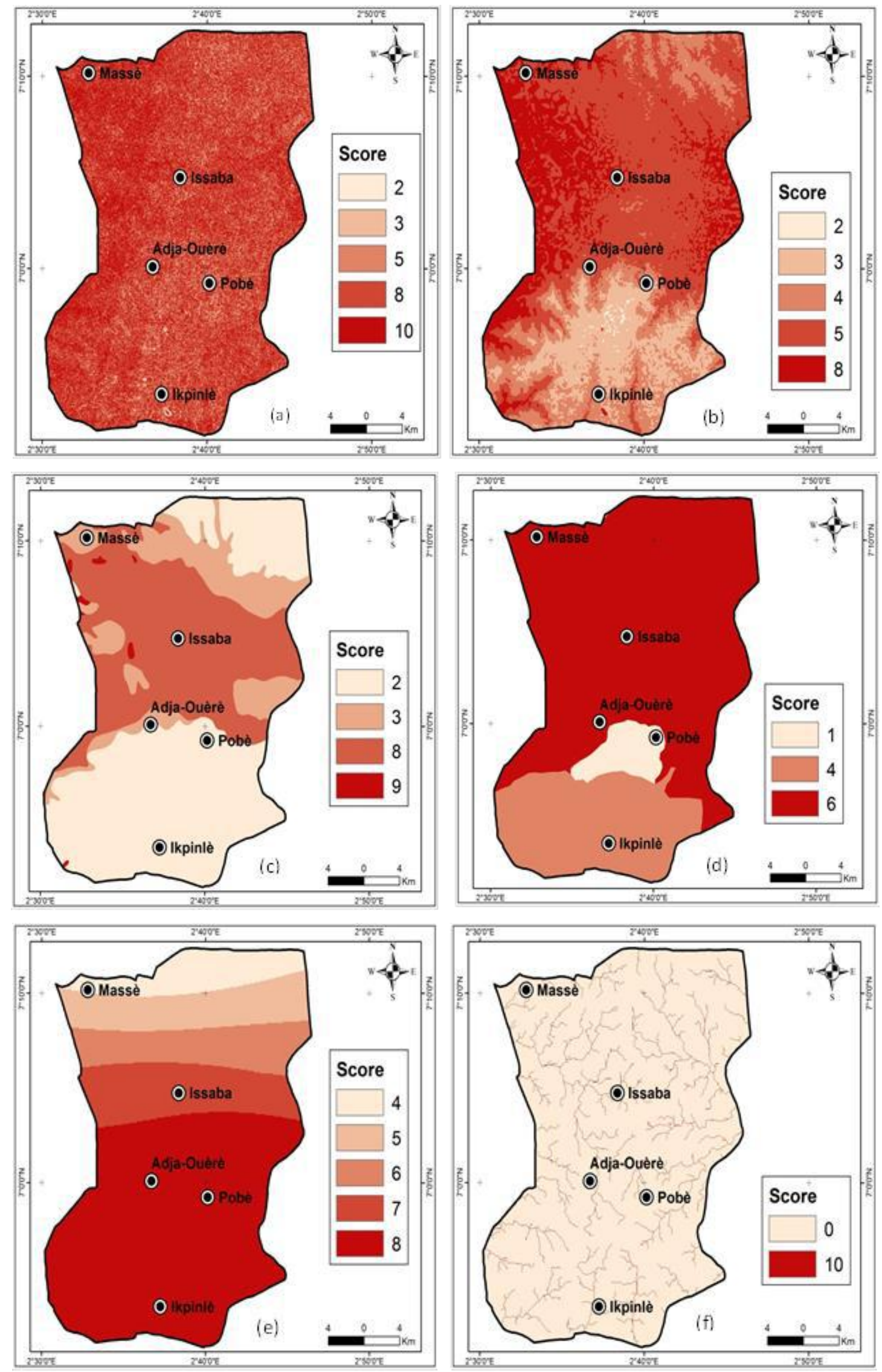

Planche 1 : Cartes critères aléa [(a) pente, (b) altitude, (c) types de sols, (d) potentialité en eau souterraine, (e) pluviométrie, (f) accumulation des eaux] 
La carte de l'aléa a été réalisée en agrégeant les différentes cartes critères par une utilisation de l'outil de superposition pondérée. La figure 3 présente la carte de l'aléa inondation dans le secteur d'étude.

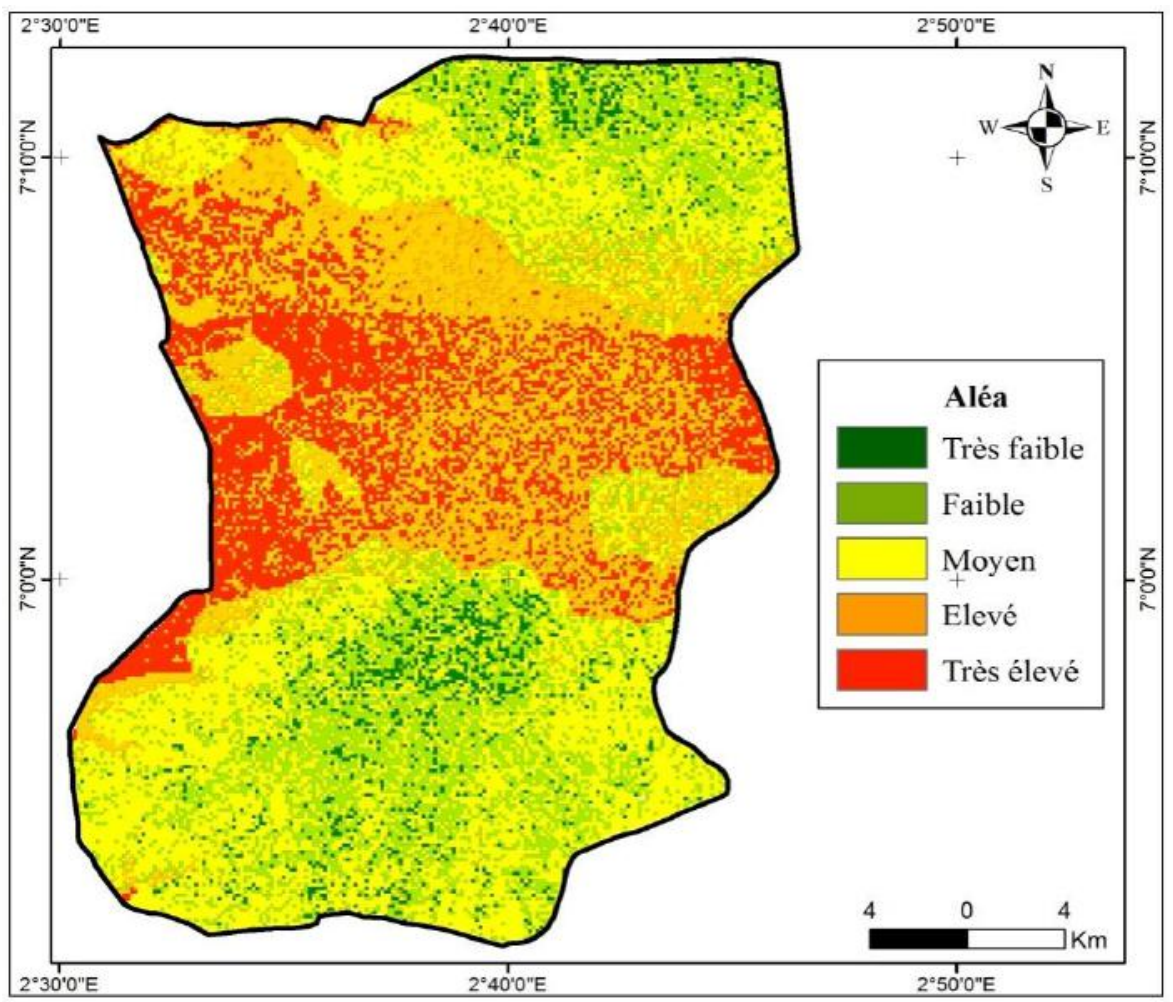

Fig.3: Carte de l'Aléa inondation

La superposition pondérée des cartes critères conduit à l'obtention de la carte de l'aléa. Cette dernière a été catégorisée en cinq classes (Très faible, Faible, Moyen, Elevé, Très Elevé).

\subsection{Cartographie générale de la vulnérabilité}

En ce qui concerne l'obtention de la carte de vulnérabilité, les cartes pondérées des différents critères sont également réalisées. L'agrégation des cartes critères (format raster) conduit à la carte de vulnérabilité. La planche 2 présente les différentes cartes critères réalisées pour la cartographie de la vulnérabilité.

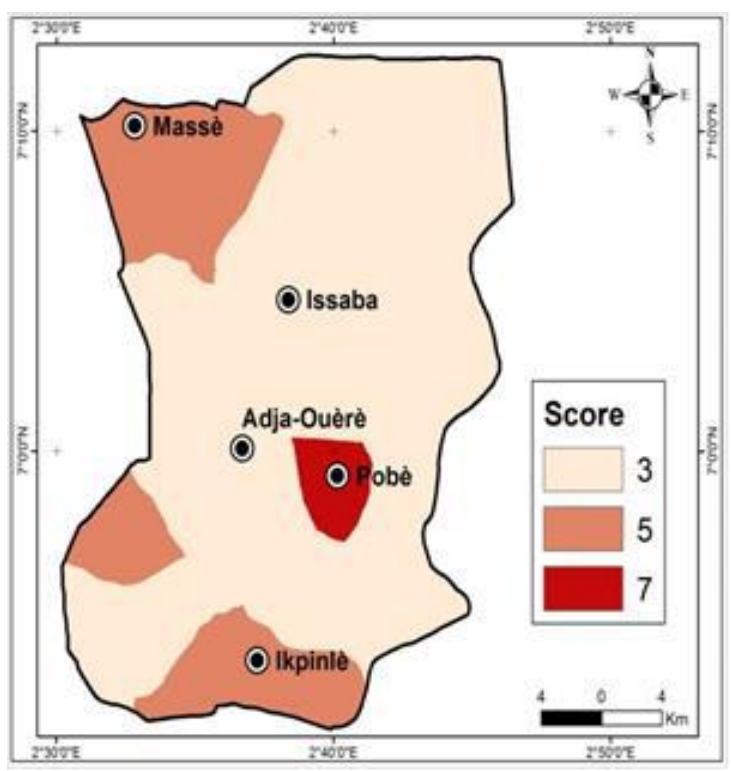

(a)

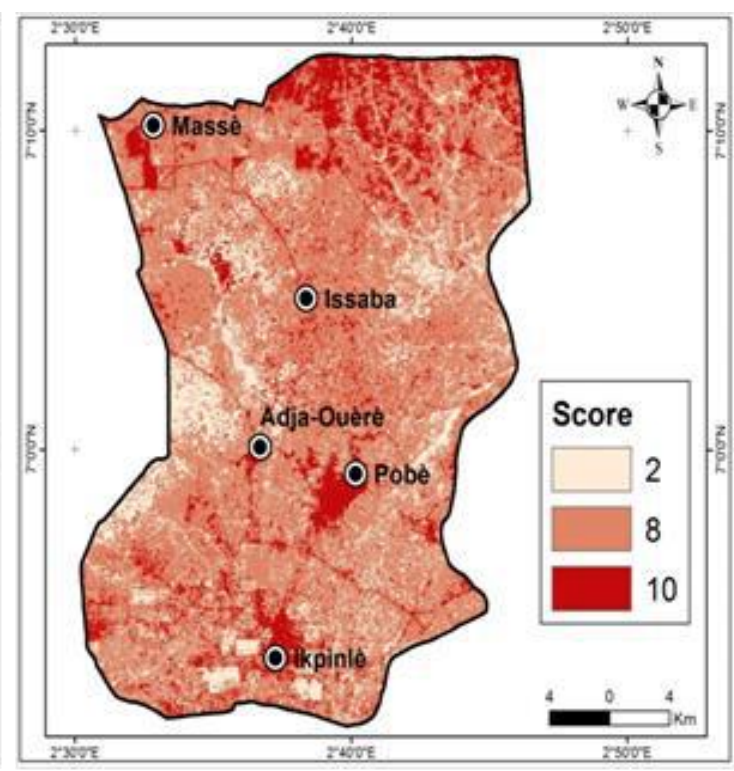

(b) 


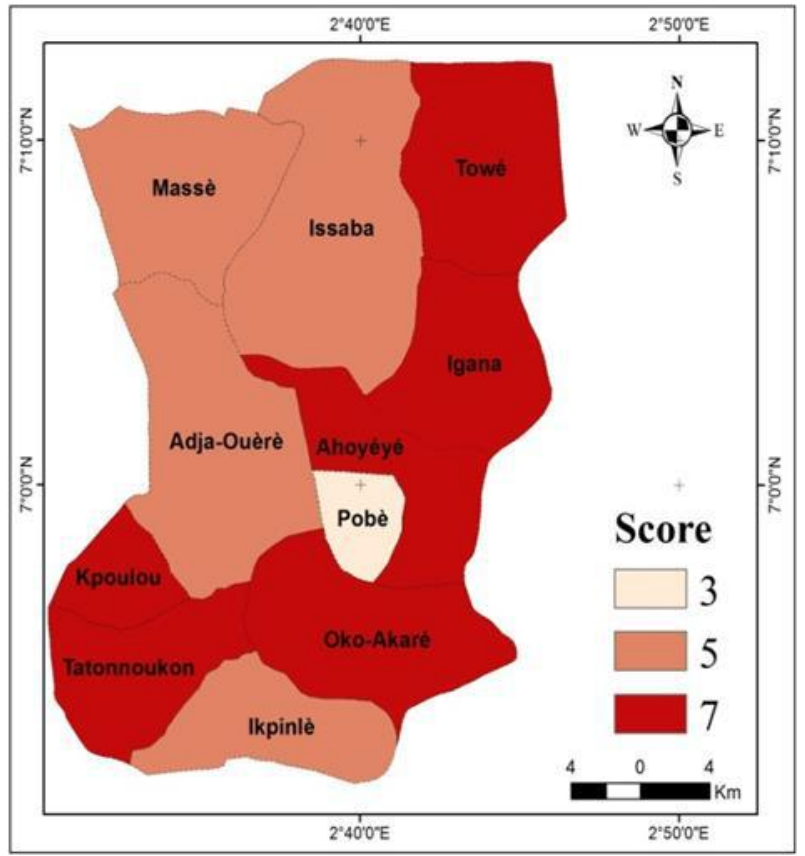

(c)

Planche 2: Cartes critères vulnérabilité [(a) densité de la population, (b) utilisation du sol, (c) degré de pauvreté]

La figure 4 présente la carte de l'aléa inondation dans le secteur d'étude obtenue après agrégation.

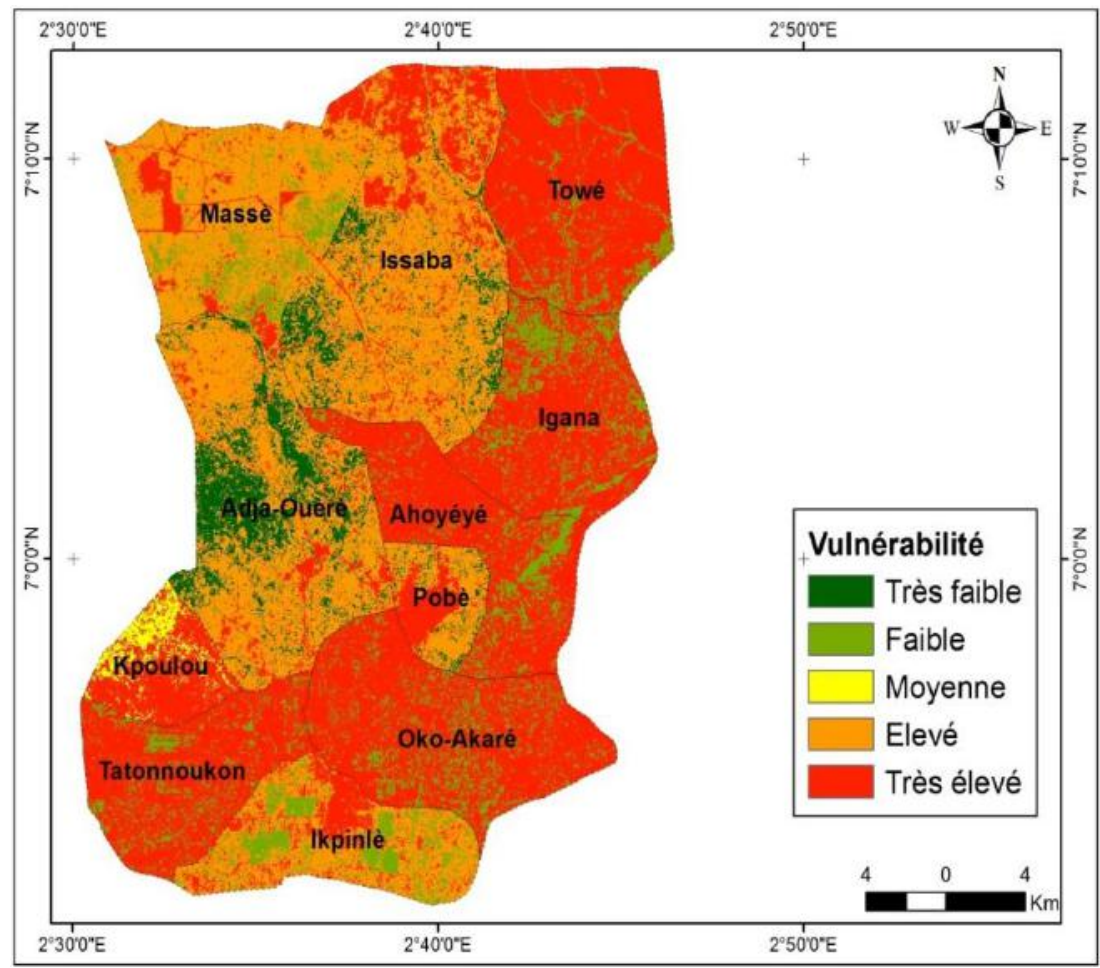

Fig.4: Carte de Vulnérabilité à l'inondation

Cette carte, obtenue à la suite de l'agrégation est catégorisée en cinq classes de vulnérabilité (Très faible, Faible, Moyenne, Elevé, Très Elevé).

\subsection{Cartographie du risque d'inondation}

La réalisation de la cartographie du risque d'inondation consiste en la superposition pondérée des cartes d'aléa et de vulnérabilité. La figure 5 présente le résultat de cette opération. 


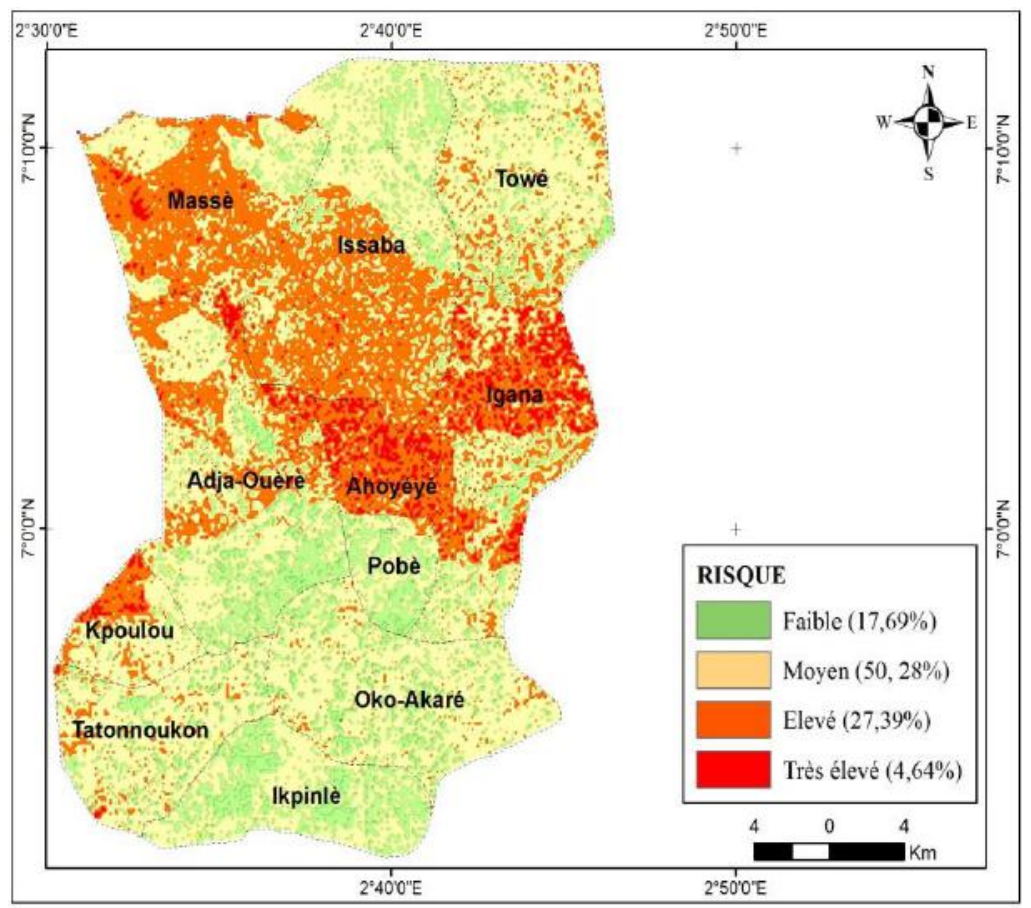

Figure 5 : Carte du risque d'inondation

Suite à la cartographie du risque d'inondation, il ressort que $17,69 \%$ de la zone d'étude présente un risque faible à l'inondation, 50,28\% un risque moyen, 27,39\% un risque élevé et 4,64\% un risque très élevé. Les arrondissements de Kpoulou, Ahoyéyé, Igana, Issaba, Adja-Ouèrè et Massè sont les plus exposés aux risques d'inondation.

\section{CONCLUSION}

L'évaluation du risque d'inondation est particulièrement importante dans la prévention des inondations puisqu'elle permet de disposer d'outils d'aides à la décision sur lesquels l'autorité peut s'appuyer. L'évaluation multicritère combinée aux SIG allie robustesse et fiabilité de la méthodologie en ce sens que l'analyse spatiale raster permet de calculer le risque d'inondation pour chaque pixel. Les critères de sélections identifiés pour la réalisation de cette étude sont subdivisés en deux types : les critères d'aléa et les critères de vulnérabilité. Les critères d'aléa sont: types de sols, la pente, la pluviométrie, l'altitude, la potentialité en eau souterraine et l'accumulation d'eau. En ce qui concerne les critères de vulnérabilité, l'utilisation du sol, le degré de pauvreté et la densité de la population ont été choisis. Les résultats obtenus à la suite de l'utilisation de l'analyse multicritère mettent en évidence quatre catégories d'espaces. Les espaces à faible risque d'inondation $(17,69 \%)$, les espaces au risque d'inondation moyen $(50,28 \%)$, les espaces aurisque élevé d'inondation $(27,39 \%)$ et les espaces au risque d'inondation très élevé $(4,64 \%)$. Suite aux enquêtes de terrain, il ressort que les phénomènes d'inondations se produisent chaque année et s'intensifient au fil du temps. Aucune mesure concrète de lutte contre l'inondation n'est menée ni par les autorités ni par les populations. Ces dernières subissent et s'adaptent à ces phénomènes en soutenant les habitations par des bois. Les pertes enregistrées annuellement s'élèvent constamment à plusieurs millions de francs CFA et parfois des décès sont enregistrées. Cette étude vise donc à mettre à la disposition du décideur un outil de prise de décision ciblée afin de mener des actions précises dans la zone d'étude en fonction du niveau de risque à l'inondation. L'intégration de critères complémentaires et l'utilisation d'un modèle numérique de terrain de haute résolution spatiale permettrait de faire gagner les futures études en précision des analyses.

\section{REFERENCES}

[1] BalzariniRaffaella, Paule-Annick Davoine et Muriel Ney : «Evolution et développement des méthodes d'Analyse spatiale multicritère pour des modèles d'aptitude: l'exemple des applications en Géosciences» Consulté le 10 octobre 2020. https://docplayer.fr/89060644-Evolution-etdeveloppement-des-methodes-d-analyse-spatialemulticritere-pour-des-modeles-d-aptitude-l-exemple-desapplications-en-geosciences.html.

[2] Ben-Mena, S. 2000. «Introduction aux méthodes multicritères d'aide à la décision ». Biotechnologie, Agronomie, Société et Environnement 4 (janvier). https://www.researchgate.net/publication/26392446_Introd 
uction_aux_methodes_multicriteres_d\%27aide_a_la_decisi on

[3] Chakhar, Salem. 2006. «Cartographie décisionnelle multicritère: formalisation et implémentation informatique ». Phdthesis, Université Paris Dauphine Paris IX. https://tel.archives-ouvertes.fr/tel-00143960.

[4] Evans, S., Gunn, N., Williams, D., \& Macdonald, M. (2008). Use of GIS in Flood Risk Mapping. https://www.semanticscholar.org/paper/Use-of-GIS-inFlood-Risk-Mapping-EvansGunn/8a05cb0981cd0292d4886327a5ba5c000cc90424?p2d $\underline{f}$

[5] Edson De Oliveira Pamplona (1999) : Avaliaçãoqualitativa de cost drivers pelométodo AHP. VI CongressoBrasileiro de Custos - São Paulo, SP, Brasil, 1999.

[6] ESRI (2016): fonctionnement de l'outil de superposition pondérée how-weighted-sum-works http://desktop.arcgis.com/fr/arcmap/10.3/tools/spatialanalyst-toolbox/how-weighted-overlay-works.htm

[7] Inondation au Bénin - Rapport d'évaluation des Besoins Post Catastrophe: Consulté le 10 octobre 2020. https://www.gfdrr.org/sites/gfdrr/files/GFDRR_Benin_PD NA_2010.pdf.

[8] Joerin, F. (1997) : Décider sur le territoire. Proposition d'une approche par utilisation de SIG et de méthodes $\mathrm{d}$ 'analyse multicritère. Thèse de Doctorat ès sciences techniques, Ecole polytechnique fédérale de Lausanne, département de génie rural, $269 \mathrm{p}$

[9] Joerin, F. (1995) : Méthode multicritère d'aide à la décision et SIG pour la recherche d'un site. Revue Internationale de Géomatique, 5.

[10] Kêdowidé, Conchita M. G. «Modélisation géomatique par évaluation multicritère pour la prospection des sites d'agriculture urbaine à Ouagadougou ». VertigO - la revue électronique en sciences de l'environnement, $\mathrm{n}^{\mathrm{O}}$ Volume 10 numéro $2 \quad$ (2 septembre 2010). https://doi.org/10.4000/vertigo.10368.

[11] Kubal C, \&Haase, Dagmar \& V, Meyer \& Scheuer, Sebastian. (2009). Integrated urban flood risk assessment Adapting a multicriteria approach to a city. Natural Hazards and Earth System Sciences. 9. 10.5194/nhess-91881-2009. Consulté le 10 octobre 2020. https://www.researchgate.net/publication/40892410_Integr ated_urban_flood_risk_assessment_Adapting_a_multicriteria_approach_to_a_city.

[12] MahsaSafaripour, MasoudMonavari, Mehdi Zare, Zahra Abedi, AlirezaGharagozlou (2012) : Flood Risk Assessment Using GIS (Case Study: Golestan Province, Iran). Pol. J. Environ. Stud. Vol. 21, No. 6 (2012), 1817 1824. http://www.pjoes.com/Flood-Risk-AssessmentUsing-GIS-r-n-Case-Study-Golestan-Province-Iran88932,0,2.html

[13] Rincon Romero, Daniela, TijjaniUsman, et Costas Armenakis. «Flood Risk Mapping Using GIS and MultiCriteria Analysis: A Greater Toronto Area Case Study ». Geosciences $8 \quad(27 \quad$ juillet 2018): 275. https://doi.org/10.3390/geosciences8080275.
[14] Saaty, Thomas L. « How to Make a Decision: The Analytic Hierarchy Process ». European Journal of Operational Research, Desicion making by the analytic hierarchy process: Theory and applications, 48, $\mathrm{n}^{\circ} 1$ (5 septembre 1990): 9-26. https://doi.org/10.1016/0377-2217(90)90057I.

[15] TAM, TzeHuey, Ab Latif Ibrahim, Muhammad Abd Rahman, et MazuraZulkifli. Flood Risk Mapping using Geospatial Technologies and Hydraulic Model. 34th Asian Conference on Remote Sensing 2013, ACRS 2013. Vol. 4, 2013.https://www.researchgate.net/publication/260593670 Flood_Risk_Mapping_using_Geospatial_Technologies_an d Hydraulic Model

[16] Wallez, Lucile (2010). «Inondations dans les villes d'Afrique de l'ouest : diagnostic et éléments de renforcement des capacités d'adaptation dans le grand Cotonou ». Maîtrise en environnement et master en Ingénierie et Management de l'Environnement et du Développement Durable, Université de Sherbrooke. http://www.usherbrooke.ca/environnement/fileadmin/sites/ environnement/documents/Essais2010/Wallez L 31-082010_.pdf

[17] Yeganeh, Nasim\&Sabri, Soheil. (2014). Flood Vulnerability Assessment in Iskandar Malaysia Using Multi-criteria Evaluation and Fuzzy Logic. Research Journal of Applied Sciences, Engineering and Technology. $8 . \quad 1794-1806.10 .19026 /$ rjaset.8.1167 https://www.researchgate.net/publication/286125907_Floo d_Vulnerability_Assessment_in_Iskandar_Malaysia_Using _Multi-criteria_Evaluation_and_Fuzzy_Logic 